\title{
Blood glucose measurement by glucometer in comparison with standard method in the diagnosis of hypoglycemia in sick neonates
}

\author{
Selvakumar $\mathbf{P}^{1}$, Vivek $\mathbf{G}^{2}$ \\ ${ }^{1}$ Dr. Selvakumar P, Associate Professor, ${ }^{2}$ Dr. Vivek G, Junior Resident, both authors are affiliated with \\ Department of Pediatrics, Thanjavur Medical College, Thanjavur, Tamil Nadu 613004, India.
}

Address for Correspondence: Dr. Selvakumar P, 180, 9 ${ }^{\mathrm{TH}}$ Cross, Arulananda Nagar Extension, Thanjavur. E-mail: drselva_kumar69@yahoo.co.in

\begin{abstract}
Hypoglycemia is a serious risk factor in neonates. The signs and symptoms are non-specific. Early diagnosis is essential to reduce the associated morbidity and mortality. Objective: To determine the efficacy and correlation of capillary and venous bedside glucose estimation using a glucometer in comparison with laboratory blood glucose analysis by glucose oxidase method in sick hypoglycemic neonates. Methods: Blood glucose estimation was done in 200 sick neonates admitted to NICU by glucometer and by glucose oxidase method in the laboratory using the same venous sample at the time of admission. In addition, glucose estimation of capillary blood was also done using the same glucometer. Statistical analysis was done by Pearson correlation. Results: The incidence of hypoglycemia in sick neonates by laboratory analysis was $15.5 \%$. When laboratory values were used as gold standard, capillary blood glucose estimates had a sensitivity of $74.19 \%$, specificity of $98.2 \%$, PPV of $88.4 \%$ and NPV of $95.4 \%$, whereas venous blood glucose estimates had a sensitivity of $93.55 \%$, specificity of $98.23 \%$, PPV of $90.62 \%$ and NPV of $98.8 \%$ in identifying hypoglycemia by glucometer in sick neonates. Conclusion: Venous and capillary blood glucose estimation by glucometer has a good sensitivity and negative predictive value in detecting hypoglycemia in sick neonates. Further, the sensitivity of glucometer using venous blood is superior to capillary sample estimates. Laboratory blood glucose estimation should still be performed if bedside venous or capillary blood glucometer values are in the hypoglycemic range.
\end{abstract}

Key words: Hypoglycemia, Glucometer, Neonates, Glucose oxidase method

\section{Introduction}

Glucose is the major energy source for the fetus and neonate. The newborn brain depends on glucose almost exclusively. Up to $90 \%$ of glucose used is consumed by the brain. Alternate fuels (e.g. ketones and lactate) are produced in very low quantities. Glucose regulating mechanisms is sluggish at birth.

Thus, the neonate is susceptible to hypoglycemia when glucose demands are increased or when exogenous or endogenous glucose supply is limited. Both preterm and term neonates are at risk of adverse neuro developmental outcome with prolonged hypoglycemia. The clinical features of neonatal hypoglycemia are non-specific and it is not possible to diagnose hypoglycemia clinically.

Manuscript received: $4^{\text {th }}$ July 2017

Reviewed: $14^{\text {th }}$ July 2017

Author Corrected: 22nd July 2017

Accepted for Publication: $29^{\text {th }}$ July 2017
Neonatal hypoglycemia is not always symptomatic. Hence frequent monitoring of blood glucose at bedside is needed in newborns.

When glucometers designed for capillary sample testing are used for blood glucose estimation using venous blood their accuracy has been questioned [1, 2]. There are concerns regarding accuracy of capillary blood glucose estimation when systemic illness coexists, where venous sampling may be more accurate [3]. In the laboratory, blood glucose estimation done using glucose oxidase method is specific and precise, because they are less affected by metabolitesand not affected by hematocrit, but the results are not immediately available. If blood sample is not delivered to the laboratory and analysis done immediately, blood glucose level will drop by $15-20 \mathrm{mg} / \mathrm{dl} /$ hour at room temperature [4, 
5]. Therefore glucose estimation is done in neonates using glucometers in the NICU. Studies have shown that glucometer values correlate well with the laboratory analysis of glucose in the normoglycemic and hyperglycemic range but are not satisfactory in the lower range $[6,7]$.

The aim of this study was to determine the efficacy and correlation of bedside capillary and venous glucose estimation by glucometer (Accu check active) in comparison with laboratory blood glucose estimation by glucose oxidase method in sick neonates.

\section{Methodology}

This hospital-based analytical cross-sectional study was conducted in the neonatal intensive care unit of a tertiary care teaching hospital in Thanjavurfrom January 2016 to July 2016. Approval from the Institute Ethics Committee was obtained prior to the conduct of the study and written informed consent was taken from the parents. There were 204 sick neonates admitted to Level 3 NICU during the study period, who were included in the study. Four neonates with $\mathrm{PCV}<40 \%$ or $>65 \%$ at admission were excluded from the study. At admission a detailed antenatal and perinatal history was taken and a complete physical examination was done.
$2 \mathrm{ml}$ of blood was drawn from a peripheral vein and three samples were taken from this at the time of admission. A bedside glucometer analysis was performed on one sample and the other two samples were sent to the clinical laboratory where PCV and blood glucose by glucose oxidase method were estimated within 30 minutes. Venous blood glucose and capillary blood glucose levels of plasma obtained from heel prick were estimated using Accu check active (Roche) glucometer.

A single glucometer calibrated and validated, following manufacturer guidelines, was used for all the neonates enrolled in the study. All bedside capillary and venous glucose estimates and laboratory glucose estimates were recorded on a standardized data sheet. The values obtained by laboratory estimation were taken as gold standard and hypoglycemia was defined as blood glucose value below $40 \mathrm{mg} / \mathrm{dl}$.

Statistical Analysis- The correlation between capillary and venous bedside glucose estimation by glucometer in comparison with laboratory blood glucose analysis was determined by Pearson correlation. Sensitivity, specificity, positive and negative predictive values were used to interpret the data. Statistical significance was set at $\mathrm{p}$ value $<$ 0.05 .

\section{Results}

Of the 200 neonates enrolled in the study, 118 were males and 82 were females with a male female ratio of 1.4:1. In our study $120(60 \%)$ were term babies,80 (40\%) were preterm babies; $139(69.5 \%)$ neonates were inborn and $34(17 \%)$ were mechanically ventilated. Of the 200 neonates screened, $31(15.5 \%)$ were hypoglycemic by laboratory glucose oxidase method.Among the 31 hypoglycemic neonates $20(64.5 \%)$ were males, $11(35.5 \%)$ were females with a male to female ratio of $2.1: 1$.Term babies were $15(48.4 \%)$, preterm were $16(51.6 \%)$, outborn were $13(41.9 \%)$ and 5(16.1\%) required mechanical ventilation among hypoglycemic neonates. Blood glucose was performed by three methods on all neonates at admission. The blood glucose values by various methods of estimation are shown in table 1.

Table-1: Blood glucose values by various methods of estimation.

\begin{tabular}{|c|c|c|c|}
\hline \multirow{2}{*}{$\begin{array}{c}\text { Blood glucose } \\
\text { levels(mg/dl) }\end{array}$} & Laboratory glucose oxidase & Glucometer capillary sample & Glucometer venous sample \\
\cline { 2 - 4 } & 31 & 26 & 32 \\
\hline$<40$ & 164 & 164 & 161 \\
\hline $40-150$ & 5 & 10 & 7 \\
\hline$>150$ & & &
\end{tabular}

Among the 31 hypoglycemic neonates detected by the laboratory method, 23(74.2\%) and $29(93.5 \%)$ were also hypoglycemic by glucometer estimates using capillary and venous blood respectively. Capillary blood glucose estimated by glucometer had a sensitivity of $74.19 \%$, specificity of $98.2 \%$, positive predictive value of (PPV) of $88.4 \%$, negative predictive value (NPV) $95.4 \%$ and accuracy of $94.5 \%$ in detecting hypoglycemia in sick 
neonates. Detection of hypoglycemia using venous blood by glucometer was found to have sensitivity of $93.55 \%$, specificity of $98.23 \%$, PPV of $90.62 \%$, NPV of $98.8 \%$ and accuracy of $97.5 \%$.

When the laboratory blood glucose was $>40 \mathrm{mg} \%$, the correlation coefficient for the lab blood glucose versus the capillary blood glucometer glucose was 0.919 and the correlation coefficient for the laboratory glucose and venous glucometer glucose was 0.928 in sick neonates. When the laboratory blood glucose was $<40 \mathrm{mg} \%$, the correlation coefficient for the lab blood glucose versus the capillary blood glucometer glucose was 0.237 and the correlation coefficient for the laboratory glucose and venous glucometer glucose was 0.216 .

\section{Discussion}

Hypoglycemia is a common complication of many neonatal problems with varied pathophysiological mechanisms. Neonates who are premature, born to diabetic mothers, large or small for gestational age are at higher risk.Yet, there is no substantial evidence to prove that there are undesirable lasting outcomes that occur at a specific level or duration of hypoglycemia. Hence the consensus is to evolve an 'Operational Threshold'. Operational threshold for hypoglycemia is defined as concentration of plasma or whole blood glucose at which a clinician should consider intervention based on the evidence currently available in literature [8]. It is presently suggested to treat neonates with a glucose concentration of less than $40 \mathrm{mg} / \mathrm{dl}[8]$.

Of the 200 sick neonates with varied symptomatology who were enrolled in the study, 31 (15.5\%) were hypoglycemic by laboratory glucose oxidase method. The incidence of hypoglycemia when determined by Accu check active glucometer was $32(16 \%)$ and $26(13 \%)$ in venous blood and capillary blood samples respectively.

Wide variations in the incidence of hypoglycemia in neonates were reported by different authors ranging from $4.8 \%$ to $41.2 \%$ [6, $9-12]$. The wide variations in incidence may be due to lack of standard definition of hypoglycemia, the time after birth when the glucose was measured, whether whole blood or serum (plasma) was used to measure the glucose concentration. Authors in various studies have provided different but variable definition of hypoglycemia : $(<30 \mathrm{mg} / \mathrm{dl})$ P.K Singhal et al.[10], $(<20 \mathrm{mg} / \mathrm{dl})$ PK Mishra and Bina Sharma [11]and $(<45 \mathrm{mg} / \mathrm{dl})$ Harish et al.[13].In our study hypoglycemia was defined as blood sugar $<40 \mathrm{mg} / \mathrm{dl}$.

In our study the incidence of hypoglycemia among term neonates was $12.5 \%$, whereas it was $20 \%$ in preterms. Sreenivasa B et al [14] detected hypoglycemia in $32.3 \%$ of term and $45.6 \%$ of preterm neonates. Arun Kumar De et al. [15] reported the incidence as $29.95 \%$ in term and $77.77 \%$ in preterm. The incidence was more common in preterm when compared with term neonates, which is comparable with other studies.

Capillary-blood-glucose monitoring by handheld glucometeris a practical solution for measuring blood glucose in neonatal intensive care units, because they are portable, cost-effective and requires minimal blood volume. Further it gives quick results, when compared with the dispatching of samples to the central laboratory. Sensitivity, specificity, positive and negative predictive values of capillary blood glucose estimates by glucometer in various studies are summarized in Table 2.

Table-2: Sensitivity, specificity and predictive values of capillary glucometer glucose estimates in various studies.

\begin{tabular}{|c|c|c|c|c|}
\hline & Sensitivity (\%) & Specificity (\%) & PPV (\%) & NPV (\%) \\
\hline Our study & 74.19 & 98.2 & 88.4 & 95.4 \\
\hline Hamid MH et al.[6] & 98 & 93 & 88 & 99 \\
\hline Sreenivasa et al.[14] & 86.17 & 99.19 & 70.28 & 99.6 \\
\hline Fatemeh Nayeri et al.[16] & 83 & 97.5 & 80 & 98 \\
\hline
\end{tabular}

In our study, the accuracy of glucometer to detect hypoglycemia in newborn using capillary-derived sample in comparison with lab glucose oxidase method was $94.5 \%$. The accuracy was $66 \%$ in Sreenivasa et al [14] study. 
Estimation of venous blood glucose using glucometer was found to have a sensitivity of $93.55 \%$, specificity of $98.23 \%$, PPV of $90.62 \%$, NPV of $98.8 \%$ and accuracy of $97.5 \%$ with statistical significant $p$ value $<0.001$, which are similar to Harish et al. [13] study. Venous blood glucose estimation using glucometers enable early initiation of treatment in sick neonates. The procedure has the advantage of not requiring capillary specimen, decreased patient discomfort and prevention of additional needle stick injury to the staff. The higher sensitivity reported in our study in detecting hypoglycemia in venous blood in comparison with capillary blood using glucometer could be attributed to inclusion of only sick neonates.

In our study, there was good correlation between laboratory blood glucose estimation and estimates of glucose using capillary or venous samples by glucometer when the blood glucose was $>40 \mathrm{mg} \%$. There was only modest correlation when the blood glucose values were $<40 \mathrm{mg} \%$, which could be attributed to the small sample size in the hypoglycemic range, which is the limitation in our study.

Bedside reagent strips (glucose oxidase), though widely used as an important point of care method, is useful for screening purpose. Low values should always be confirmed by formal laboratory analysis [17]. However, treatment may be initiated based on the results of the reagent strips. Further studies with large sample size are required to determine the accuracy of venous and capillary blood glucose estimation using glucometer in sick hypoglycemic neonates.

\section{Conclusion}

Venous and capillary blood glucose estimation by glucometer has a good sensitivity and negative predictive value in detecting hypoglycemia in sick neonates. In our study, the sensitivity of venous derived bedside blood glucose was superior to capillary glucometer estimates in detecting hypoglycemia.

Laboratory blood glucose estimation should still be performed if bedside venous or capillary blood glucometer values are in the hypoglycemic range or the results are likely to significantly alter clinical management.

Funding: Nil, Conflict of interest: None initiated, Perission from IRB: Yes

\section{References}

1. Funk DL, Chan L, Lutz N, Verdile VP. Comparison of capillary and venous glucose measurements in healthy volunteers. Prehosp Emerg Care. 2001 Jul-Sep;5(3):275-7.

2. Boyd R, Leigh B, Stuart P. Capillary versus venous bedside blood glucose estimations. Emerg Med J. 2005 Mar; 22 (3):177-79. doi: 10.1136/emj. 2003.011619.

3. Atkin SH, Dasmahapatra A, Jaker MA, Chorost MI, Reddy S. Fingerstick glucose determination in shock. Ann Intern Med. 1991 Jun 15;114 (12): 1020-4.
4. Jain A, Aggarwal R, Jeevasanker M, Agarwal R, Deorari AK, Paul VK. Hypoglycemia in the newborn. Indian J Pediatr. 2008 Jan;75(1):63-7.

5. Elusiyan JB, Adeodu OO, Adejuyigbe EA. Evaluating the validity of a bedside method of detecting hypoglycemia in children. Pediatr Emerg Care. 2006 Jul; 22(7):488-90.

6. Hamid MH, Chishti AL, Maqbool S. Clinical utility and accuracy of a blood glucose meter for the detection of neonatal hypoglycemia. J Coll Physicians Surg Pak. 2004Apr;14(4);225-8.

7. Ngerncham S, Piriyanimit $\mathrm{S}$, Kolatat $\mathrm{T}$, Wongsiridej P, Inchgarm L, Kitsommart R, Vutrapongwatana P, Jeerapaet K. Validity of two point of care glucometers in the diagnosis of neonatal hypoglycemia. Indian Pediatr. 2012 Aug; 49 (8):621-5. Epub 2011 Jan 17.

8. Hay Jr WW, Raju TNK, Higgins RD, et al. Knowledge gaps and research needs for understanding and treating neonatal hypoglycemia: workshop report from Eunice Kennedy Shriver National Institute of Child Health and Human Development. J Pediatr. 2009Nov;155(5):6127.doi:10.1016/j.jpeds.2009.06.044.

9. Cornblath M, Hawdon JM, Williams AF, Aynsley-Green A, Ward-Platt MP, Schwartz R, Kalhan SC. Controversies regarding definition of 
Original Research Article

neonatal hypoglycemia: suggested operational thresholds. Pediatrics. 2000 May;105(5):1141-5.

10. Singhal PK, Singh M, Paul VK, Deorari AK, Ghorpade MG, Malhotra A. Neonatal hypoglycemia --clinical profile and glucose requirements. Indian Pediatr.1992 Feb;29(2):167-71.

11. Misra PK, Sharma B. Hypoglycemia in newborns - a prospective study. Indian Pediatr. 1977 Feb;14(2):129-32.

12. Anderson S, Shakya KN, Shrestha LN, Costello AM. Hypoglycaemia: a common problem among uncomplicated newborn infants in Nepal. J Trop Pediatr. 1993 Oct;39(5):273-7.

13. Harish J, Srinivas HA, Soumya A. Comparative study of glucometer and laboratory glucoseoxidase method for the estimation of blood glucose levels inneonates: jemds.2015 Feb; 4(16): 2652-63.doi:10. 14260 /jemds/2015/383.
14. Sreenivasa B, Kumar GV. Comparative study of blood glucose levels in neonates using glucometer and laboratory glucose oxidase method. CurrPediatr Res. 2015;19(1\&2);29-32.

15. De AK,Biswas R, Samanta M, Kundu CK. Study of blood glucose level in normal and low birth weight newborns and impact of early breast feeding in a tertiary care center. Ann Nigerian Med. 2011;5 (2): 53-8.doi:10.4103/0331-3131.92951.

16. Nayeri F, Shariat M, Mousavi Behbahani HM, Dehghan P, Ebrahim B. Blood glucose measurement by glucometer in comparison with standard method in diagnosis of neonatal hypoglycemia. Acta Med Iran. 2014;52(8):619-22.

17. Ho HT, Yeung WK, Young BW. Evaluation of point of care devices in the measurement of low bloods glucose in neonatal practice. Arch Dis Child Fetal Neonatal Ed. 2004 Jul;89(4):F356-F359.

\section{How to cite this article?}

Selvakumar P, Vivek G. Blood glucose measurement by glucometer in comparison with standard method in the diagnosis of hypoglycemia in sick neonates. J PediatrRes.2017;4(07):469-473.doi:10.17511/ijpr.2017.i07.06. 